\title{
Sleep Disorders in Perimenopausal Women and Metabolic Syndrome, is that True?
}

\author{
Pilnik Susana*, Belardo Maria Alejandra, Cavanna Malena, De Nardo Barbara and Starvaggi Agustina \\ Department of Gynecology, Italiano de Buenos Aires Hospital, Argentina
}

Received: 彗June 22, 2018; Published: 阱July 02, 2018

*Corresponding author: Pilnik Susana, Department of Gynecology, Italiano de Buenos Aires Hospital, Argentina

\begin{abstract}
Sleep disorders (SD) are a common complaint in women going through menopause transition. It is well known that there is a close relationship between SD and one of the main menopausal symptoms: vasomotor symptoms (VMS). To acknowledge this association is essential when investigating menopausal transition and SD. The Study of Women's Health across the Nation (SWAN), shows that the prevalence of sleep disturbance increases with age. The prevalence in perimenopausal and postmenopausal women varies from $39 \%$ to $47 \%$ and $35 \%$ to $60 \%$ respectively. Although both menopausal transition and aging increase sleep disturbances, etiology in menopausal women is probably multifactorial. A decline in the levels of reproductive hormones and melatonin, and the complex interaction among these hormones can significantly contribute to sleep problems, poor concentration, fatigue and decreased quality of life. On the one hand menopause nearly adversely affects all components of metabolic syndrome (MS) and on the other hand the cumulative long-term effects of deprived sleep have been associated with many cardio metabolic consequences including hypertension, obesity, and impaired glucose metabolism. Management strategies should be taken into account to help women ameliorate their night sleep, in order to prevent future complications and to improve their quality of life (QoL).
\end{abstract}

Keywords: Sleep disorders; Menopause; Vasomotor symptoms; Metabolic syndrome

Abbreviations: SD: Sleep Disorders; VMS: Vasomotor Symptoms; SWAN: Study of Women's Health Across the Nation; MS: Metabolic Syndrome; QoL: Quality of Life; OSA: Obstructive Sleep Apnea; FSH: Follicle Stimulating Hormone; IR: Insulin Resistance; DBT: Type2 Diabetes; CVD: Cardio Vascular Disease; HPA: Hypothalamus-Pituitary-Adrenal; AHI: Apnea Hypopnea Index; GABA: GammaAmino Butyric Acid;

\section{Introduction}

Despite the fact that most adults need at least eight hours sleep every night to be well rested, not everyone achieves it. Not getting enough sleep for a long time might cause health problems. According to the Third Edition of the International Classifications of Sleep Disorders, both insomnia and obstructive sleep apnea (OSA) are identified as part of the seven major categories of SD [1]. SD are highly prevalent in general population, for example, insomnia is estimated to affect up to $30 \%$ of people [2]. The latter is more prevalent in women than in men and several studies, which evaluated SD with subjective scales, indicated that women in general population have worse sleep quality than men [3]. Prevalence of SD increases dramatically from $30 \%$ in premenopausal women to approximately $50 \%$ in peri and postmenopausal women [4]. The Study of Women's Health across the Nation (SWAN), a multiethnic female sample of 12,603 , found that $38 \%$ of women aged $40-55$ referred sleep difficulties, significantly related to menopause. The
SWAN study also found that the prevalence of SD was related to ethnic origin, varying from $28 \%$ in Japanese women to $40 \%$ in Caucasians [5]. It is important to mention the fact that compared to Asian women; Latin American mid-aged women display a higher prevalence of VMS [6].

A total of 6079 women aged 40-59 of 11 Latin American countries were invited to fill out validated scales. Insomnia and poor sleep quality were highly prevalent in this mid-aged female sample in which the influence of age and the menopause was only modest and rather linked to menopausal symptoms already occurring since the premenopausal [6]. Referring to our experience in Argentina, we analyzed the prevalence of SD in postmenopausal women using validated scales, and concluded that insomnia presented a prevalence of $22.2 \%$ [7]. The causes of SD are multifactorial, the high prevalence in peri and postmenopausal women, shows that VMS, changing reproductive hormone levels, circadian rhythm 
abnormalities, mood disorders, coexisting medical conditions, and lifestyle (such as troublesome drinking, drug use: hypnotics and hormone therapy) may play an important role. Higher educational level related to less insomnia and better sleeps quality [6] (Figure 1).

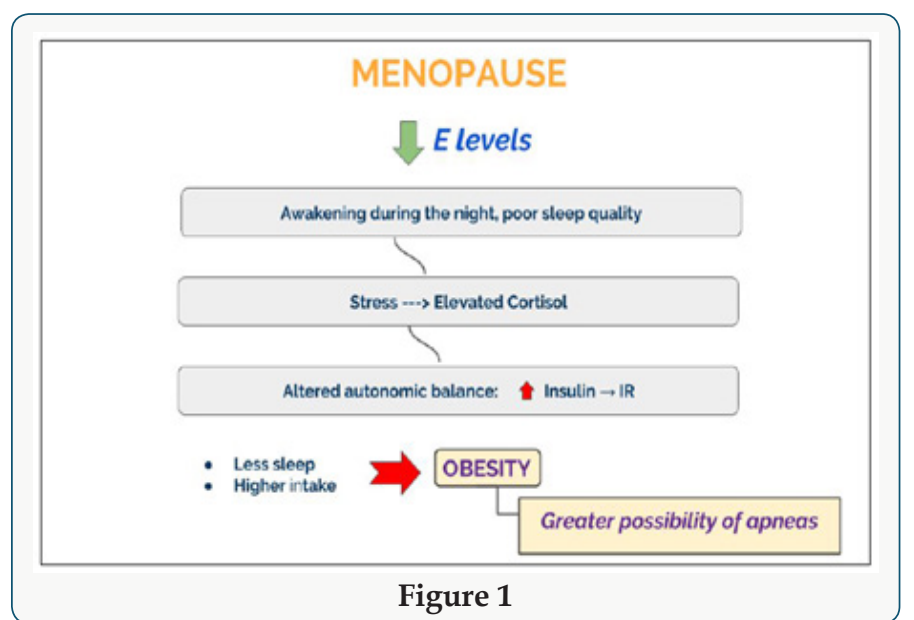

\section{Estradiol, Melatonin and Changes in Sleep}

At a physiological level, the decreasing levels of estradiol and increasing follicle stimulating hormone (FSH) levels may interfere with melatonin secretion and other circadian hormones [8]. Melatonin levels decrease particularly during the perimenopausal period. In addition, aging may be an important consideration. While women age, their circadian rhythm changes and decreases melatonin secretion resulting in shortened sleep duration and early morning awakening. Reduced levels and atypical secretion patterns of melatonin have been also observed in OSA [9]. However other studies have found that the best predictor of SD in menopause was the history of previous SD [10]. Taking into account that changes in estradiol levels produce sympathetic activation, we might consider as a hypothesis that this contributes to the initiation of hot flashes by narrowing the thermo neutral zone [6]. Anxiety and depression have also been linked to estradiol fluctuations. Four distinct neurotransmitter systems have been shown to be affected during the menopause: gamma amino butyric acid, serotonin, nor adrenaline and dopamine.

It is of interest to mention that dopamine and serotonin have been implicated in the regulation of sleep, and estrogendependent changes in serotonergic neuronal transmission during menopausal transition have been cited as a possible cause for sleep changes, mood fluctuations and memory loss. Good sleep quality is required for both good health and QoL [6]. Polysomnography was used to evaluate hot flashes and their correlation with SD in a group of healthy premenopausal women who received leuprolide (gonadotropin-releasing hormone agonist) to rapidly induce menopause. By isolating the effect of hot flashes on women's sleep without confusing with age, this experimental model showed that nocturnal VMS correlate with increased sleep fragmentation. The number of women studied was small but consistently suggests the specific contribution of VMS to sleep interruption [11].

\section{Key points [12]}

a. Around 40 to $60 \%$ of women reports sleep impairment during menopause.

b. Etiology of SD in menopausal women is probably multifactorial.

c. VMS and depressive symptoms could be the most important risk factors for impaired sleep quality.

d. Menopausal transition has been documented as an important hallmark for increased about 3.4 times the risk of sleep disorders in postmenopausal women compared with premenopausal women.

e. $\quad 20 \%$ of women in this category say they have a difficult time sleeping due to hot flashes or night sweats at least a few nights each week.

\section{Health Consequences of Sleep Deprivation}

Evidence has emerged in recent years that sleep plays an important role in modulating weight gain, neuro endocrine signaling, and consequently insulin and glucose homeostasis [13]. Health consequences of SD involve vascular, metabolic and neuropsychiatric outcomes, including hypertension, heart disease, arrhythmia, stroke, heart failure, insulin resistance (IR) and type-2 diabetes (DBT). Sleep also impacts negatively on mood. Insomnia, for example, is co morbid with depression and anxiety [14]. The longitudinal Penn State Cohort study of 1344 adults followed for a mean of 16.6 years found that odds of metabolic syndrome (MS) associated all-cause and cardiovascular disease (CVD) mortality were greater for individuals who slept $<6$ vs. $\geq 6$ hours each night. The authors found that the effect modification of sleep duration was observed mainly on the elevated blood pressure and glucose dysregulation components of the MS [15]. Circadian rhythm desynchrony has likewise been shown to adversely affect glucose metabolism. Studies suggests that superimposing circadian desynchrony with sleep restriction leads to a decompensation of $\beta$-cell function characterized by severely impaired insulin secretion [16]. Another mechanism that has been proposed is that sleep loss, especially slow wave sleep, exerts adverse effects on glucose homeostasis by stimulating the hypothalamus-pituitary-adrenal (HPA) axis and sympathetic activity [17].

\section{Metabolic Syndrome and Sleep Disorders: Which Comes First?}

Considering the prevalence of SD in the general population, the epidemiological and pathophysiological links between MS, incident DBT and CVD, the association of OSA with MS and IR is of critical importance [18].

Which comes first? This supposition is based on the following premises [18]:

a. Many sleep apneas do not have structural abnormalities in their upper airways and, vice versa, many patients with 
narrow upper airways owing to anatomical abnormalities do not have sleep apnea.

b. Obesity increases the risk of OSA

c. Sleep apnea is very frequent in disorders in which IR is a primary pathophysiologic abnormality, e.g. polycystic ovary syndrome, in which IR is the strongest predictor of the presence of sleep apnea.

d. IR, by releasing growth factors, may lead to soft tissue edema and tissue proliferation in the neck

e. Some metabolic abnormalities are associated with excessive daytime sleepiness.

f. Pro-inflammatory mediators (known to be elevated in OSA) are also independently associated with excessive daytime sleepiness

g. Anti-inflammatory interventions have the potential to decrease sleepiness and apnea hypopnea index (AHI).

There are several connection points between MS and SD. For example, every $10 \mathrm{~kg}$ gained OSA risk twofold and with an increase in waist or hip circumference by $13-15 \mathrm{~cm}$ OSA risk fourfold. Visceral fat especially predicts OSA and significantly correlates with apnea hypopnea index. On the other hand, there may be a reciprocal relationship between obesity and OSA, such that not only does obesity increase the risk of OSA, but also that sleep apnea may predispose to weight gain and obesity. Indeed, patients with newly diagnosed OSA have difficulty losing weight and, in fact, are predisposed to excessive weight gain, far more than are evident in similarly obese control subjects proven to be free of OSA [18]. Association between OSA and dyslipidemia is causal and independent of other confusing factors (especially obesity), it is noteworthy that OSA lowers HDL, raises total cholesterol/HDL ratio and triglycerides [18].

Abundant evidence supports the role of enhanced daytime sympathetic activity, which results not only from a carry-over effect from the nocturnal events, but may also be related to chemoreceptor resetting and tonic 'normoxic' chemoreceptor activation. Other potential contributing mechanisms that have been at baseline described in OSA include baroreflex and endothelial dysfunction. Irrespective of the exact mechanism of action, it appears that OSA can, in and of itself, lead to elevated blood pressure and thus contribute to yet another component of the MS [18]. Evidence has accumulated over the last few years, however, to support the

concept that OSA may be directly related to IR, independent of obesity and other anthropometric measures, in both obese and nonobese subjects. OSA is also characterized by a pro-inflammatory state and elevated cytokine levels (e.g. tumor necrosis factor- $\alpha$, TNF- $\alpha$ ). Furthermore, OSA may induce oxidative stress owing to repetitive episodes of intermittent hypoxia, and increased oxidative stress has been shown to be an important pathogenic mechanism of
IR [18]. OSA-related hypercoagulability may be related to increased platelet aggregability, increased haematocrit, elevated fibrinogen levels and increased blood viscosity [18].

\section{Sleep and Cortisol}

Sleep has a modest but clearly detectable effect on the HPA axis since it exerts an inhibitory action on the secretion of cortisol. Sudden changes in sleep rhythm induce a disruption in cortisol daily rhythm. Both the OSA and awakenings during the night would determine an activation of the axis generating an increase in cortisol levels leading to disruption in the daily rhythm. If this persists chronically, it could have metabolic, cognitive and psychiatric consequences [19]. As a consequence of the autonomic disbalance, insulin levels raise leading to IR. Moreover, sleep deprivation is accompanied by an increase in food intake that could bring to obesity and greater possibility of apneas.

\section{Sleep and Inflammation}

Many pro-inflammatory cytokines regulate sleep and physiological functions. Restriction between 25 to $50 \%$ of a normal sleep cycle alters these mediators of inflammation [20]. Studies suggest that sleep loss is associated with an increased monocyte production of IL-6 and TNF-alpha. These variations in this circulating

markers of inflammation are associated with the development of cardiovascular events, hypertension, obesity and type 2 diabetes [21]. Summarizing, menopause comprehend a period of high risk for SD. A marked decrease in the production of estradiol, which results in VMS, such as hot flashes and night sweats, is related to awakening during the night and poor sleep quality. Also circadian rhythm changes and decreases melatonin secretion. These hormonal changes seem to affect sleep directly. Insufficient sleep has been associated with increased risk of obesity, disorders of glucose, insulin homeostasis, and MS.

\section{Treatment}

\section{Non - Pharmacological Approaches}

The FDA suggests several tips for sleep hygiene [22]:

a. Go to bed and get up at the same time each day.

b. Avoid caffeine, nicotine, beer, wine and liquor four to six hours before bedtime.

c. Don't exercise within two hours of bedtime.

d. Don't eat large meals within two hours of bedtime.

e. Don't nap later than 3 p.m.

f. Sleep in a dark, quiet room that isn't too hot or cold for you.

g. If you can't fall asleep within 20 minutes, get up and do something quiet. 
h. Wind down in the 30 minutes before bedtime by doing something relaxing.

Advice about psycho education and sleep hygiene includes the following Strategies [23]:

a. Relaxation training: The progressive muscle relaxation, autogenic training, imagery training, meditation help to get a better sleep. Yoga and Therapeutic Massage are a helpful option.

b. Stimulus control therapy: Consists in a set of behavioral instructions designed to re-associate the bed/bedroom with sleep and to re-establish a consistent sleep-wake schedule.

c. Sleep restriction therapy: Points to curtail the time in bed to the actual amount of sleep being achieved.

d. Cognitive Behavioral Therapy: Is a psychological method designed to identify, challenge and change misconceptions about sleep and faulty beliefs about insomnia.

\section{e. Exercise}

\section{Pharmacological Approaches}

Furthermore, dietary supplements (melatonin and tryptophan), sedative hypnotics (benzodiazepine and non-benzodiazepine hypnotics) and antidepressants (selective serotonin and nor epinephrine reuptake inhibitors: SSRIs and SNRIs) are an important complementary option to non-pharmacological directions [23].

\section{What about Hormone Therapy?}

Primary pharmacological treatments for menopause related VMS including insomnia revolve around replacing the diminished levels of estrogen and/or progesterone levels. The added estrogen contributes to sleep through metabolizing nor epinephrine, serotonin and acetylcholine, which consequently increases REM

cycles. On the other hand, progesterone stimulates benzodiazepine receptors, causing the release of gamma-amino butyric acid (GABA), a sedating neurotransmitter that can potentially facilitate sleep [23]. Micronized progesterone at a dose of $300 \mathrm{mg} /$ night significantly reduces VMS and thus, adding its sedative effect, improves the quality of sleep [24].

\section{Conclusion}

Identifying women at risk for developing SD during menopausal transition is essential in preventing the consequences of sleep deprivation. Good sleep quality is required for both, good health and QoL. Sleep restriction contribute to increase the prevalence of MS in postmenopausal women. Early and comprehensive

treatment plays a central role in the prevention of cardiovascular disease.

\section{References}

1. Michael J Sateia (2014) International Classification of Sleep Disorders ( $3^{\text {rd }}$ Edn.): Highlights and Modifications. CHEST 146(5): 1387-1394.
2. Tao M, Sun D, Shao H, Li C, Teng Y (2015) Poor sleep in middle-aged women is not associated with menopause per se. Braz J Med Biol Res 49(1): e4718.

3. Zhang B, Wing YK (2006) Sex differences in insomnia: a meta-analysis. Sleep 29(1): 85-93.

4. Carrier J, Kazue S, Deurveilher S, Drogos L, Cyr-Cronier J, et al. (2017) Sex differences in age-related changes in the sleep-wake cycle. Front Neuroendocrinol 47: 66-85.

5. Kravitz H, Ganz P, Bromberger J, Powell L, Sutton-Tyrrell K, et al. (2003) Sleep difficulty in women at midlife: a community survey of sleep and the menopausal transition. Menopause 10(1):19-28.

6. Blumel J, Cano A, Mezones-Holguin E, Baron G, Bencosme A, et al. (2012) A multinational study of sleep disorders during female mid-life. Maturitas 72(4): 359-366.

7. Belardo A, Valiensi S, Tutzer M, Bozza L, Starvaggi A (2014) Evaluation of sleep quality in postmenopausal women. Congreso AAPEC.

8. Shechter A, Boivin D (2010) Sleep, Hormones, and Circadian Rhythms throughout the Menstrual Cycle in Healthy Women and Women with Premenstrual Dysphoric Disorder. Int J Endocrinol 2010: 259345.

9. Jehan S, Giardin J, Ferdinand Z, Evan A, Pandi-Perumal S, et al. (2017) Sleep, Melatonin, and the Menopausal Transition: What Are the Links? Sleep Sci 10(1): 11-18.

10. Smith R, Flaws J, Mahoney M (2018) Factors associated with poor sleep during menopause: results from the Midlife Women's Health Study. Sleep Med 45: 98-105.

11. Bianchi M, Kim S, Galvan T, White D, Joffe H (2016) Nocturnal Hot Flashes: Relationship to Objective Awakenings and Sleep Stage Transitions. J Clin Sleep Med. Jul 15 12(7): 1003-1009.

12. Lampio L, Saaresranta T, Engblom J, Polo O, Polo-Kantola P (2016) Predictors of sleep disturbance in menopausal transition. Maturitas 94: 137-142.

13. Koren D, Taveras E (2018) Association of sleep disturbances with obesity, insulin resistance and the metabolic syndrome. Metabolism 84: 67-75.

14. Schmid S, Hallschmid M, Schultes B (2015) The metabolic burden of sleep loss. Lancet Diabetes Endocrinol 3(1): 52-62.

15. Fernandez-Mendoza J, He F, La Grotte C, Vgontzas A, Liao D, et al. (2017) Impact of the Metabolic Syndrome on Mortality is Modified by Objective Short Sleep Duration. J Am Heart Assoc 6(5): pii: e005479.

16. Scheer F, Hilton M, Mantzoros C, Shea S (2009) Adverse metabolic and cardiovascular consequences of circadian misalignment. Proc Natl Acad Sci U S A106(11): 4453-4458.

17. Kuem S, Lin K, Insop S (2012) Stress and Sleep Disorder. Exp Neurobiol 21(4): 141-150.

18. Wolk R, Somers V (2006) Sleep and the metabolic syndrome. Exp Physiol 92(1): 67-78.

19. Balbo M, Leproult R, Van Cauter E (2010) Impact of Sleep and Its Disturbances on Hypothalamo-Pituitary-Adrenal Axis Activity. Int J Endocrinol 2010: 759234.

20. Mullington J, Simpson N, Meier-Ewert H, Haack M (2010) Sleep Loss and Inflammation. Best Pract Res Clin Endocrinol Metab. Oct 24(5): 775-784.

21. Irwin M, Olmstead R, Carroll J (2016) Sleep Disturbance, Sleep Duration, and Inflammation: A Systematic Review and Meta-Analysis of Cohort Studies and Experimental Sleep Deprivation. Biol Psychiatry. Jul 1 80(1): 40-52.

22. FDA U.S Food \& Drug (2017) Sleep Problems. Office of Women's Health. 
23. Joshua Z, Sooyeon A, Dowdle C, Nowakowski S (2015) Treatment of Insomnia, Insomnia Symptoms, and Obstructive Sleep Apnea During and After Menopause: Therapeutic Approaches. Curr Psychiatry Rev 11(1): 63-83.
24. The NAMS 2017 Hormone Therapy Position Statement Advisory Panel. The 2017 hormone therapy position statement of The North American Menopause Society. Menopause 24(7): 728-753.

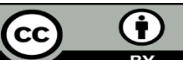

This work is licensed under Creative Commons Attribution 4.0 License

Submission Link:

Submit Article

DOI: $10.32474 /$ OAJRSD.2018.01.000109

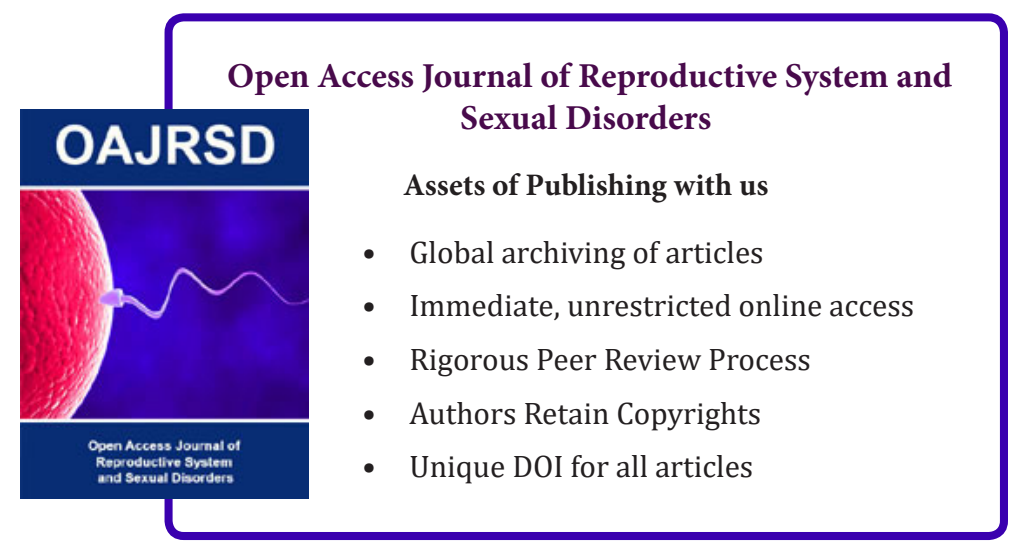

